\title{
LINEAR REPRESENTATIONS OF CERTAIN COMPACT SEMIGROUPS $\left({ }^{1}\right)$
}

\author{
BY \\ D. R. BROWN AND MICHAEL FRIEDBERG
}

\begin{abstract}
In this paper we initiate the study of representation theory of compact, not necessarily commutative, uniquely divisible semigroups. We show that a certain class of semigroups are all topologically isomorphic to real matrix semigroups. The proof utilizes a group embedding theorem and the standard results on homomorphisms of Lie groups into matrix groups.
\end{abstract}

1. Introduction. A semigroup $S$ is (uniquely) divisible provided for each $x \in S$, and each positive integer $n$ there exists a (unique) $y \in S$ such that $y^{n}=x$. The basic properties of uniquely divisible semigroups have been developed in [5], [10], and [11]. Representation theory of these objects has been examined in [3], [4], [16], and [17]. Related material occurs in [13].

In this paper we initiate the study of representation theory of compact, not necessarily commutative, uniquely divisible semigroups. We shall be concerned exclusively with faithful (real) finite-dimensional linear representations. Clearly, objects admitting such representations are necessarily finite dimensional. For earlier work involving representations of (nongroup) topological semigroups, see [2] and [9].

$\S \S 2$ and 3 are preparatory in nature. In $\S 4$ we show that a certain class of semigroups are all topologically isomorphic to real matrix semigroups. The conditions such semigroups satisfy are restrictive. However, for mitigation the reader is asked to recall the following:

(1) the structure of real matrix semigroups is quite special, and

(2) the theorem proved is one in which no linear structure is presumed upon the semigroup investigated.

The matrix semigroup

$$
\left\{\left(\begin{array}{ll}
x & y \\
0 & 1
\end{array}\right): x, y, x+y \in[0,1]\right\}
$$

is an example of the class of semigroups we study here; it has served us as a model for the proof and it may aid the reader as motivation for the hypotheses. Higher

Received by the editors July 7, 1970.

AMS 1969 subject classifications. Primary 2205; Secondary 2090, 2260.

Key words and phrases. Divisible semigroup, linear representation.

(1) Research by the authors supported in part by NSF Grant GP 11889.

Copyright (C) 1971, American Mathematical Society 
dimensional analogues may be constructed by taking the convex hull of the $(n \times n)$ identity matrix with the left trivial semigroup

$$
\left\{\left(\begin{array}{cccc}
0 & \cdots & 0 & x_{1} \\
0 & \cdots & 0 & x_{2} \\
\vdots & & \vdots & \\
0 & \cdots & 0 & x_{n-1} \\
0 & \cdots & 0 & 1
\end{array}\right): x_{i} \in[0,1], i=1,2, \ldots, n-1\right\}
$$

It is conjectural as to whether every $n$-dimensional semigroup of the type studied in $\S 4$ is embeddable in this example.

In connection with the cancellation hypothesis of Theorem 4.1, we note that in [1] it has been shown that if $M(S)$ is a 1-cell, and $S$ satisfies all other hypotheses of Theorem 4.1, then $S \backslash M(S)$ does have cancellation. Further, no higher dimensional examples of this type are known in which $S \backslash M(S)$ fails to have cancellation.

Finally, we adhere to the semigroup terminology of [12]. The semigroup of $n \times n$ real matrices will be denoted by $M_{n}(R) ; \mathrm{Gl}_{n}(R)$ will represent the subgroup of invertible elements in $M_{n}(R)$. The minimal ideal of $S$ will be $M(S)$, and the set of idempotents of $S$ will be $E(S)$. We shall use $\square$ for the empty set. For any $e \in E(S)$, Core $(e)=\{x \mid x e=e=e x\}$.

We are indebted to several people for their comments concerning various aspects of this material. In particular, H. Cook, J. D. Lawson, and J. W. Stepp have aided us by their insights.

2. A group embedding theorem. In this section, we prove a theorem on the (topological) group embeddability of a certain class of topological semigroups. Our methods differ from those of Rothman [22] and Christoph [6] in that the semigroup in question is first algebraically embedded in a group, then a topology is introduced into the group such that the group is topological and the embedding function is a homeomorphism. In order to accomplish this, we shall call upon two well-known theorems which we state without proof. A semigroup is right reversible provided every pair of principal left ideals has a nonempty intersection.

TheOREM A (ORE-REeS) [7]. A right reversible semigroup $S$ with two-sided cancellation is embeddable in a group $G$ such that $G=S^{-1} S$. The group $G$ is, to isomorphism, unique, and is called the group of right quotients of $S$.

TheOREM B (R. ElLis) [8]. Let $G$ be a group having the property that the underlying space of $G$ admits a topology $\mathscr{V}$ such that $(G, \mathscr{V})$ is a locally compact Hausdorff space. If right and left translation functions are continuous in this topology, then $(G, \mathscr{V})$ is a topological group.

We now state the embedding theorem alluded to above. 
THEOREM 2.1. Let $S$ be a topological semigroup defined on a closed subset of Euclidean n-space $\left(E^{n}\right)$ having nonempty interior (relative to $\left.E^{n}\right)$. If $S$ is right reversible and has two-sided cancellation, and if right translation functions are homeomorphisms, then $S$ is embeddable in a Lie group $G$ of dimension $n$ in such a way that $S^{0}$, the interior of $S$ relative to $E^{n}$, is open in $G$.

We defer the proof of 2.1 in order to establish a lemma useful both here and in the sequel.

LEMMA 2.2. Under the hypotheses of 2.1, $S^{0}$ is a two-sided ideal of $S$, and $G=\left(S^{0}\right)^{-1} \cdot\left(S^{0}\right)$.

Proof. Let $x \in S$ and $y \in S^{0}$. Then $x y \in x S^{0}$, and this set is open in $E^{n}$ by invariance of domain (see [15], for example). Hence $x S^{0} \subseteq S^{0}$. This proves that $S^{0}$ is a right ideal, and the proof that $S^{0}$ is a left ideal is clearly similar. For the second claim, let $g \in G, g=x^{-1} y, x, y \in S$. Since $S^{0} x \subseteq S^{0}$, there exist $s, t$ in $S^{0}$ such that $x=s^{-1} t$. Thus both $x$ and $y$ belong to the set $\left(S^{0}\right)^{-1}\left(S^{0}\right)$, and to complete the proof it suffices to show that this set is a subsemigroup of $G$. This follows from the chain of inclusions $\left(S^{0}\right)^{-1}\left(S^{0}\right)\left(S^{0}\right)^{-1}\left(S^{0}\right) \subseteq\left(S^{0}\right)^{-1}(S)^{-1}(S)\left(S^{0}\right) \subseteq\left(S^{0}\right)^{-1}\left(S^{0}\right)$.

Proof of Theorem 2.1. In light of prior remarks, we denote by $G$ the group of right quotients of $S$ and proceed to topologize $G$. Denote by $\varphi$ the previously anonymous embedding function carrying $S$ into $G$. Let $\mathscr{U}$ be the family of all open subsets of $S^{0}$, and let $\mathscr{V}=\{x U y: U \in \varphi(\mathscr{U}), x, y \in G\}$. To see that $\mathscr{V}$ is a subbasis for a topology on $G$, note that for any $z \in G$, and any $x \in S^{0}, z=x^{-1} x z \in x^{-1} S^{0} z$. Clearly, in this topology on $G$, left and right translation functions are homeomorphisms of $G$ onto itself, so that $G$ is homogeneous in this topology.

We show next that $\varphi$ restricted to $S^{0}$ is a homeomorphism. It is immediate from the definition of the topology on $G$ that $\varphi$ is an open function. Next, let $z \in S$, let $p, q, r, s \in \varphi(S), U \in \varphi(\mathscr{U})$ such that $\varphi(z)$ is an element of $p^{-1} q U r^{-1} s$. By Lemma 2.2 , there exist elements $a, b$ in $\varphi\left(S^{0}\right)$ such that $p \varphi(z) s^{-1}=a^{-1} b$. It follows directly that $b r \in a q U$. By continuity of the semigroup multiplication in $S$, there exist $W \in \varphi(\mathscr{U})$ such that $b \in W$ and $W r \subseteq a q U$. Thus, $a p \varphi(z)=b s \in W s$, and $W s \in \varphi(\mathscr{U})$ by invariance of domain. Again by continuity of multiplication in $S$, there exists $V \in \varphi(\mathscr{U})$ such that $\varphi(z) \in V$ and $a p V \subseteq W s$. Direct computation yields $V \subseteq p^{-1} q U r^{-1} s$. This establishes the continuity of $\varphi$ at $z$ with respect to subbasic neighborhoods of $\varphi(z)$ and the fact that $\varphi$ is one-to-one yields continuity of $\varphi$ at $z$ for arbitrary open sets containing $\varphi(z)$. Hence $\varphi$ is a homeomorphism on $S^{0}$. Since $S^{0}$ is locally compact and $\varphi\left(S^{0}\right)$ is open in $G$, and $G$ is homogeneous, we have that $G$ is locally compact.

To prove that $G$ is Hausdorff, fix $a \in \varphi\left(S^{0}\right)$. It is clear that $a$ can be separated from any other element of $\varphi\left(S^{0}\right)$. Hence let $b \in G \mid \varphi\left(S^{0}\right)$. By Lemma 2.2, $b=x^{-1} y$ for some pair $x, y$ in $\varphi\left(S^{0}\right)$. Hence $x a$ and $y$ are distinct elements of $\varphi\left(S^{0}\right)$. By continuity in $S$ and the fact that $S^{0}$ is a Hausdorff space, there exist $V, W \in \varphi(\mathscr{U})$ such 
that $a \in W, y \in V$, and $x W \cap V=\square$. It follows that $W$ and $x^{-1} V$ are disjoint members of $\mathscr{V}$, containing $a$ and $b$, respectively. Since $a$ has now been shown to be separated from any other element of $G$, and $G$ is homogeneous, we have that $G$ is a Hausdorff space. By Theorem B, $G$ is a topological group in the topology induced by $\mathscr{V}$, and, since $S^{0}$ is locally Euclidean, we have that $G$ is locally Euclidean and hence a Lie group [19].

To complete the proof of Theorem 2.1, it remains to be shown that $\varphi$ is a homeomorphism on all of $S$. The fact that $\varphi$ is continuous on all of $S$ follows just as in the argument for $S^{0}$, the exception being that the $V$ obtained now is open only in $S$ rather than in $S^{0}$. To prove that $\varphi$ is an open map, let $W$ be an open set in $S$, let $z \in W$, and let $a$ be an arbitrary fixed element of $S^{0}$. By hypothesis, $W a$ is open in $S a$. Since $W a \subseteq S^{0}$, there exists an open set $U \in \mathscr{U}$ such that $W a=S a \cap U$. Hence $\varphi(z) \in \varphi(U) \varphi(a)^{-1}, \varphi(U) \varphi(a)^{-1} \in \mathscr{V}$, and $\varphi(U) \varphi(a)^{-1} \cap \varphi(S)=\varphi(W)$. This completes the proof of the theorem.

3. Theorems on uniquely divisible semigroups. We set forth in this section certain basic theorems about compact uniquely divisible semigroups which we shall need in the remainder of the paper. Throughout this section, $S$ will denote a compact uniquely divisible semigroup. As in previous papers in this area (see, for example, [3], [10], and [11]), [x] will denote the closure of the rational powers of $x$. In the case that $S$ has trivial maximal groups and $x$ is a nonidempotent, it is well known that $[x]$ is topologically isomorphic to the unit interval $[0,1]$ under real number multiplication [10]. The following result has been reported by J. D. Lawson but does not appear in print.

THEOREM 3.1. Let $S$ be such that $E(S)=M(S) \cup\{1\}$. Then $S \backslash M(S)$ is a subsemigroup of $S$.

Proof. By passing to the Rees quotient of $S$ modulo $M(S)$, it suffices to consider the case in which $S$ has a zero and prove that $S$ does not have zero divisors. To this end, let $x, y$ in $S$ be such that $x y=0$ and assume $x$ is not zero. Clearly $(y x)^{2}=0$, whence by uniqueness of roots $y x=0$. Thus $0=x y=\left(x^{1 / 2} x^{1 / 2}\right) y=x^{1 / 2}\left(x^{1 / 2} y\right)$, so that $\left(x^{1 / 2} y\right) x^{1 / 2}=0$. Therefore $\left(x^{1 / 2} y\right)^{2}=0$, and hence $x^{1 / 2} y=0$. Inductively, $x^{1 / 2^{n}} y$ $=0$. Since the cluster points of the sequence $\left\{x^{1 / 2^{n}}\right\}$ are known to form a subgroup of $H(1),[14]$, it follows that $y=0$, which completes the proof.

Theorem 3.2. Let $S$ have $E(S)=\{0,1\}$, and let $H(1)=1$. Let $P^{*}$ be the nonnegative real numbers, and let $g: S \backslash\{0\} \times P^{*} \rightarrow S \backslash\{0\}$ be defined by $g(x, r)=x^{r}$. Then $g$ is continuous, and if $\left\{\left(x_{\lambda}, r_{\lambda}\right)\right\}$ is a net in $S \backslash\{0\} \times P^{*}$ such that $\left\{x_{\lambda}\right\}$ converges to $x \neq 1$ and $\left\{r_{\lambda}\right\}$ increases without bound, then $\left\{g\left(x_{\lambda}, r_{\lambda}\right)\right\}$ converges (in $\left.S\right)$ to 0 .

Proof. This is Theorem 1.3 of [3]. The following result is of use in $\$ 4$.

THeOREM 3.3. Let $S$ have $E(S)=M(S) \cup\{1\}$, and let $H(1)=1$. Then for each pair of distinct elements $x, y \in S \backslash M(S)$, there exists $h: S \rightarrow[0,1]$ such that $h$ is 
continuous, $h(1)=1, h(M(S))=0, h(x) \neq h(y)$, and for each $z \in S \backslash E(S), h$ restricted to $[z]$ is a topological isomorphism.

Proof. Clearly, we may shrink $M(S)$ by use of the Rees quotient and assume that $S$ has a zero. In this case the $\mathscr{J}$-relation on $S$ defined by $a \mathscr{J} b$ if and only if $S a S \subseteq S b S$ is known to be a partial order with closed graph [12], and we replace $a \mathscr{J} b$ by $a \leqq b$ for the remainder of the proof. Assuming $x \leq y$, by a well-known theorem of Nachbin [20], there exists a continuous function $f: S \rightarrow[0,1]$ which is order preserving and satisfies $f(x)=1, f(y)=0$. Let $\left\{r_{n}\right\}_{n=1}^{\infty}$ be an enumeration of the positive rational numbers such that $r_{1}=1$. Define $g(z)=\sum_{n=1}^{\infty}\left(1 / 2^{n}\right) f\left(z^{r}\right)$; it is easily checked that $g$ is a continuous function from $S$ into $[0,1]$ such that $g(1)=1$, $g(0)=0$. Furthermore, $g(x)>\frac{1}{2} f(x)=\frac{1}{2}$, whereas $g(y)=\frac{1}{2}$. Now, let $z \in S \backslash E(S)$ and let $r$ and $s$ be real numbers satisfying $0 \leqq s<r$, so that for each $n, s r_{n}<r r_{n}$. It follows that $f\left(z^{r r_{n}}\right) \leqq f\left(z^{s r_{n}}\right)$ for each $n$, and hence that $g\left(z^{r}\right) \leqq g\left(z^{s}\right)$. Should equality hold, we would have $f\left(z^{r r_{n}}\right)=f\left(z^{s r_{n}}\right)$ for each $n$, whence, by Theorem 3.2, $f\left(z^{r p}\right)=f\left(z^{s p}\right)$ for each nonnegative real number $p$. Fix any such $p$ and let $q=p / r$; then $f\left(z^{r q}\right)=f\left(z^{s q}\right)$, so that $f\left(z^{t p}\right)=f\left(z^{p}\right)$, where $t=s / r$. By iteration, $t$ may be replaced by $t^{k}$ for any positive integer $k$. Since $t<1$, it follows that $f\left(z^{p}\right)=f\left(z^{0}\right)=f(1)=1$. Thus, for any positive integer $n, f\left(z^{n}\right)=1$; however $\left\{z^{n}\right\}$ converges to zero in $S$, so that the continuity of $f$ is contradicted. Thus $g\left(z^{r}\right)<g\left(z^{s}\right)$.

Since $g$ is continuous, for each $z \in S \backslash E(S)$ we have that there exists a (unique) positive real number $r$ such that $g\left(z^{r}\right)=\frac{1}{2}$. Now, define $h_{0}: S \rightarrow[0, \infty]$ by $h_{0}(1)=0$, $h_{0}(0)=\infty$, and $h_{0}(z)=1 / r$ if $z \in S \backslash E(S)$ and $g\left(z^{r}\right)=\frac{1}{2}$. We omit the verification of the continuity of $h_{0}$, which is straightforward but tedious.

Next, let $z \in S \backslash E(S)$ and fix $s$, a positive real number. If $h_{0}(z)=1 / r$, then $g\left(z^{r}\right)=\frac{1}{2}$, so that $g\left(\left(z^{s}\right)^{r / s}\right)=\frac{1}{2}$, whence $h_{0}\left(z^{s}\right)=s / r=s h_{0}(z)$. Finally, since $g(x)>\frac{1}{2}$ and $g(y) \leqq \frac{1}{2}$, we have $h_{0}(x)<1 \leqq h_{0}(y)$. The desired function $h$ is now defined by $h(0)=0, h(z)$ $=\exp \left(-h_{0}(z)\right)$ otherwise, and the requisite properties are easily checked. The final theorem of this section is a generalization of a theorem in [4].

THEOREM 3.4. Let $S$ have $E(S)=M(S) \cup\{1\}$, let $M(S)$ consist of left zero elements only ( $e f=e$, all $e, f \in M(S))$, and for each $e \in M(S)$, assume Core $(e)$ is a commutative subsemigroup of $S$. If $S \backslash M(S)$ has left cancellation, then $S \backslash M(S)$ has right cancellation.

Proof. Suppose $a, x, y \in S \backslash M(S)$ and $x a=y a$; set $u=a^{1 / 2} x a^{1 / 2}, v=a^{1 / 2} y a^{1 / 2}$. It follows directly that $u^{2}=v u, v^{2}=u v$. Then $u^{3}=(v u) u=v u^{2}=v^{2} u$, and, recursively, $u^{n+1}=v^{n} u$. Let $e, f \in M(S)$ such that $\left\{u^{n}\right\}$ converges to $e$ and $\left\{v^{n}\right\}$ converges to $f$. Then, by continuity of multiplication, $e=f u=f$, so that $u$ and $v$ must commute. We now have $u^{2}=v u=u v=v^{2}$, whence $u=v$. Invoking left cancellation, we have $x a^{1 / 2}=y a^{1 / 2}$. Inductively, it follows that $x a^{1 / 2^{n}}=y a^{1 / 2^{n}}$; hence, as in the proof of 3.1, we have $x=y$.

4. Faithful linear representations of certain semigroups. We are now prepared to state the main result of the paper. 
THEOREM 4.1. Let $S$ be a compact semigroup such that $E(S)=M(S) \cup\{1\}, M(S)$ consists of left zero elements, $M(S)$ is homeomorphic to an $n-1$ cell, and for each $e \in M(S)$, Core $(e)$ is topologically isomorphic to $[0,1]$ under real number multiplication. If $S \backslash M(S)$ admits left cancellation, then $S$ is topologically isomorphic to a semigroup of $n \times n$ real matrices in such a way that $S \backslash M(S)$ is embedded in $\mathrm{Gl}_{n}(R)$ and $M(S)$ is embedded in the rank one idempotents.

We defer the beginning of the proof in order to state several basic properties of Lie groups that will be used in the sequel. For proofs of these facts, the reader is referred to [19]. We assume $n>1$ henceforth.

If $G$ is an $n$-dimensional Lie group, then $G$ admits a continuous homomorphism $\psi$ into $\mathrm{Gl}_{n}(R)$ such that kernel $(\psi)=$ Center $(G)$. In particular, if $G$ has trivial center, then $\psi$ is a continuous isomorphism of $G$ into $\mathrm{Gl}_{n}(R)$. The standard construction leading to the definition of $\psi$ is accomplished by defining addition, scalar multiplication, and a metric on $\mathscr{L}$, the set of one parameter groups of $G$ in such a way that $\mathscr{L}$ becomes an $n$-dimensional real vector space. For each $g \in G$, an operator $A_{g}$ is defined on $\mathscr{L}$ by $A_{g}(x(t))=g x(t) g^{-1}$. The homomorphism $\psi$ is then defined by $\psi(g)=A_{g}$. The family of operators $\left\{A_{g}\right\}$ can then be assigned matrices by choosing any basis in $\mathscr{L}$. There is also now a continuous local homeomorphism defined from $\mathscr{L}$ into $G$, called the exponential function, by $\exp (x)=x(1)$. We shall not be involved with either the Lie product in $\mathscr{L}$ or any of the other analytic properties possessed by $G$.

Proof of Theorem 4.1. The argument is accomplished by putting together a sequence of lemmata indexed by the alphabet. We first establish certain elementary consequences of the hypotheses. Clearly, $S$ is connected.

(A) The group of units of $S$ is trivial. For, if $g \in H(1)$, then define $f: M(S) \rightarrow M(S)$ by $f(e)=g e$. Since $M(S)$ is a cell, by the fixed point theorem of Brouwer there exists $b \in M(S)$ such that $g b=b$. Since $M(S)$ consists of left zero elements, $b g=b$, so that $g \in$ Core $(b)$, whence $g=1$.

(B) The semigroup $S$ is uniquely divisible. If $x$ is a nonidempotent, then the powers of $x$ must converge to an idempotent $e \in M(S)$, and $x \in$ Core (e) [18]. Hence $S$ is divisible. If $b$ and $c$ are both $n$th roots of $a$, then clearly all three of these elements belong to the core of the same idempotent in $M(S)$. It follows now that $b=c$.

(C) The semigroup $S$ is homeomorphic to an $n$-cell, and $M(S)$ is a subset of the boundary $n-1$ sphere of $S$. For any $x \in S \backslash E(S)$, let $e_{x}$ be the idempotent in $M(S)$ which is the limit of the powers of $x$. Let $Q$ be the space obtained by shrinking $M(S) \times\{1\}$ out of $M(S) \times[0,1]$, and let $\mathscr{N}$ be the natural map from $M(S) \times[0,1]$ onto $Q$. It is well known that $Q$, as the cone over an $n-1$ cell, is topologically an $n$-cell. In order to map $S$ onto $Q$, let $h$ be any of the functions guaranteed by Theorem 3.3 from $S$ onto $[0,1]$. Define $\tau: S \rightarrow Q$ by $\tau(x)=\mathscr{N}\left(\left(e_{x}, h(x)\right)\right)$ if $x \neq 1$ and $\tau(1)$ $=\mathscr{N}(M(S) \times\{1\})$. The function $\tau$ is clearly well defined, one-to-one, and onto, and 
maps $M(S)$ into the boundary of $Q$. To see that $\tau$ is continuous, let $\left\{x_{\alpha}\right\}$ be a net in $S$ converging to $x$. If $x \neq 1$, it will be sufficient to show that $\left\{e_{x_{\alpha}}\right\}$ converges to $e_{x}$. Let $\left\{e_{x_{\alpha}}\right\}$ cluster to $t \in M(S)$. For each $\alpha$, we have $x_{\alpha} e_{x_{\alpha}}=e_{x_{\alpha}}$, so that $x t=t$. Hence $x^{n} t=t$ for each positive integer $n$, so that $e_{x} t=t$. Since $e_{x} t=e_{x}$, also, we have $t=e_{x}$. It is now immediate that $\tau\left(x_{\alpha}\right)$ converges to $\tau(x)$. In the case that $x=1$, the continuity of $h$ is sufficient to guarantee the continuity of $\tau$.

(D) The set $S \backslash M(S)$ is a subsemigroup of $S$ having two sided cancellation. This follows directly from Theorems 3.1 and 3.4.

We have now established that $S$ has the following properties: $S$ is a compact, connected uniquely divisible semigroup defined on an $n$-cell such that $E(S)$ $=M(S) \cup\{1\}$. Further, $M(S)$ is an $n-1$ cell, contained in the boundary of $S$, consisting entirely of left zero elements, and for each $e \in M(S)$, Core $(e)$ is topologically isomorphic to $[0,1]$ with real multiplication. Finally, $H(1)=\{1\}$. We will denote the subsemigroup $S \backslash M(S)$ by $T$. We shall have reason to deal with both the interior of $S$ (as a cell) and the interior of $M(S)$ as a cell, and we shall use $S^{0}$, and $M(S)^{0}$ respectively for these sets. This should not cause confusion to the reader, as the ordinary concept of set interior will not be brought into use throughout the proof. Note that $S^{0} \subseteq T$.

(E) The semigroup $T$ satisfies the hypotheses of Theorem 2.1 and is therefore embeddable in a Lie group of dimension $n$ in such a way that the set $S^{0}$ remains open. That right translation functions in $T$ are homeomorphisms follows from the fact that right translation functions in $S$ are homeomorphisms; this, in turn, follows from right cancellation in $T$ together with the fact that $M(S)$ consists of left zero elements. It remains to show that $T$ is right reversible. To this end, let $x$ and $y$ be elements of $T$. It must be shown that $T x \cap T y \neq \square$. Note that $T x=S x \mid M(S)$, so that, topologically, $T x$ and $T y$ are $n$-cells with a boundary $n-1$ cell removed. Let $e \in M(S)^{0}$, and let $U$ be an open set containing $e$ such that $U \subseteq M(S)^{0} \cup S^{0}$. Then $e \in U x \cap U y \subseteq S x \cap S y$, and $U x \cap U y$ is an open set in $S$. Since $S$ is an $n$-cell, it follows that $U x \cap U y$ is $n$-dimensional [15], and hence cannot be a subset of $M(S)$. Therefore we have $T x \cap T y \neq \square$, which shows that $T$ is right reversible. We denote the Lie group $T^{-1} T=\left(S^{0}\right)^{-1}\left(S^{0}\right)$ by $G$, and the embedding map of $T$ into $G$ by $\varphi$.

(F) If $x, y \in T \backslash\{1\}$ such that $x y=y x$, then (in $S$ ) $[x]=[y]$. This follows from the equation $x^{n} y^{n}=y^{n} x^{n}$, which proves $e_{x}=e_{x y}=e_{y x}=e_{y}$. Hence $x, y \in$ Core $\left(e_{x y}\right)$, which is topologically isomorphic to $[0,1]$, and thus $[x]=[y]$.

(G) There exists a continuous isomorphism of $G$ into $\mathrm{Gl}_{n}(R)$. By the remarks at the beginning of this section, it suffices to show that $G$ has trivial center to prove the function $\psi$ is one-to-one. This follows immediately from (F). Now any choice of basis in $\mathscr{L}$ will induce an isomorphism of $G$ into $\mathrm{Gl}_{n}(R)$. We permit any such function to retain the name $\psi$, and note that we now have that the function $\psi \varphi$ is a continuous isomorphism of $T$ into $M_{n}(R)$, the semigroup of $n \times n$ real matrices under matrix multiplication, and $\psi \varphi(T) \subseteq \mathrm{Gl}_{n}(R)$. The balance of the 
proof is concerned with the extension of $\psi \varphi$ to all of $S$ (for the right choice of basis in $\mathscr{L}$ ). The primary problem is to extend $\psi \varphi$ to $M(S)^{0}$.

(H) The set $\exp ^{-1}\left(\varphi\left(S^{0}\right)\right)$ is open in $\mathscr{L}$ and hence contains a basis for $\mathscr{L}$, which we denote by $\left\{x_{i}\right\}, i=1, \ldots, n$. This is immediate from the openness of $\varphi\left(S^{0}\right)$ in $G$ and the continuity of the exponential function, together with the fact that $\varphi\left(S^{0}\right) \subseteq \exp (\mathscr{L})$ by the unique divisibility of $T$.

(I) Let $z \in T \backslash\{1\}$ and suppose $z \in$ Core (e). Then $e \in M(S)^{0}$ if and only if $z \in S^{0}$. Although this is the form of the result we need, we prove the alternate theorem that $e$ belongs to the (cell) boundary of $M(S)$ if and only if $z$ belongs to the (cell) boundary of $S$, from which the former statement follows. For any positive integer $n$, the map $x \rightarrow x^{n}$ is a homeomorphism of $S$ onto itself and hence carries the boundary of $S$ back onto itself. Since $M(S)^{0}$ is open in the boundary of $S$ and $\left\{z^{n}\right\}$ converges to $e$, we have that $e$ is in the boundary of $M(S)$ if $z$ is in the boundary of $S$. Conversely, suppose that $e$ belongs to the boundary of $M(S)$. To prove that $z$ belongs to the boundary of $S$, it suffices to find $y \in T \backslash\{1\}$ such that $y \in$ Core $(e)$ and $y$ is in the boundary of $S$; for then we have all rational powers of $y$ in the boundary of $S$, whence $[y]$ will be a subset of the boundary of $S$. By the conditions on Core $(e)$, it will then follow that Core $(e)$ is a subset of the boundary of $S$, whence $z$ belongs to the boundary of $S$. To produce the desired element $y$, let $\left\{y_{n}\right\}$ be a sequence of elements of $T$ converging to $e$ such that each $y_{n}$ belongs to the boundary of $S$. Let $U$ and $V$ be disjoint open subsets of the boundary of $S$ such that $M(S) \subseteq U$ and $1 \in V$. Since each set $\left[y_{n}\right]$ is an arc meeting $M(S)$ and containing 1 , we may select a sequence $\left\{q_{n}\right\}$ such that $q_{n} \in\left[y_{n}\right] \backslash(U \cup V)$. Let $\left\{q_{n}\right\}$ cluster to $y$, and note that $y$ is in the boundary of $S$ and $y \in T \backslash\{1\}$. To prove $y \in$ Core (e), it suffices to show $y e=e$. Let $\left\{q_{k}\right\}$ be a subsequence of $\left\{q_{n}\right\}$ which converges to $y$, and note $\left\{y_{k}\right\}$ still converges to $e$. Let $e_{k}$ denote the element of $M(S)$ such that $y_{k} \in$ Core $\left(e_{k}\right)$; the sequence $\left\{e_{k}\right\}$ can then be factored as $\left\{y_{k} e_{k}\right\}$ to see that it also converges to $e$. We now have $y e=\lim _{k}\left\{q_{k} e_{k}\right\}=\lim _{k}\left\{e_{k}\right\}=e$. This completes the argument for (I).

(J) Let $x \in T$. Then $x M(S)^{0} \subseteq M(S)^{0}$. Clearly $x M(S)^{0} \subseteq M(S)$; by invariance of domain it suffices to show that if $e, f \in M(S)^{0}$ and $x e=x f$, then $e=f$. Since $S x$ is a sub- $n$-cell of $S$ containing $M(S)$ and $e \in M(S)^{0}$, there exists $y \in$ Core $(e) \cap T x$. Let $y=t x$; then $y f=t x f=t x e=y e=e$. Again, there exists $z \in \operatorname{Core}(f) \cap T x$. If $z=s x$, then $z e=f$. Hence $y z e=y f=e$, so that $y z \in \operatorname{Core}(e)$. It follows that $y(y z)$ $=(y z) y=y(z y)$. By cancellation of $y$ from the left, $y z=z y$; by part $(\mathrm{F}),[y]=[z]$ and hence $e=f$.

(K) For any $x \in S, x S \subseteq S x$; in particular, if $x \in T$, then $x T \subseteq T x$. If $x \in M(S) \cup\{1\}$, this is clear; the latter claim will follow from the former by application of Theorem 3.1. Hence let $x \in T$ and pick $y \in S^{0}$. Denote by $A$ the complement of $M(S)$ in the boundary of $S$; from the fact that right translation by $x$ is a homeomorphism of $S$ onto $S x$, we have that the cell boundary of $S x$ $=A x \cup M(S)$. It is well known [21] that the set $x[y]$ is an arc in $S$ with endpoints 
$x$ and $x e_{y}$. By (I) and (J), $x e_{y} \in M(S)^{0}$. Since right multiplication by $x$ leaves $M(S)$ fixed elementwise, the set $S x \backslash \bar{A} x$ is open not only in $S x$ but also in $S$, and contains the elements $x e_{y}$. Hence there exists a positive real number $M$ such that $x y^{r} \in S x \mid \bar{A} x$ for all $r>M$. Now suppose $x y \notin S x$. Let $(a, b)$ be the maximal open interval of nonnegative real numbers such that $x y^{r} \notin S x$ for all $r \in(a, b)$. Clearly, $b \leqq M<\infty$, so that $x y^{b}$ is defined. Moreover, $x y^{b}$ belongs to the boundary of $S x$, and hence to $A x$. Let $t \in A$ such that $x y^{b}=t x$. Then, for each positive integer $n, x\left(y^{b}\right)^{n}=t^{n} x$, whence, by continuity, $x e_{y}=e_{t} x=e_{t}$. But $x e_{y} \in M(S)^{0}$, whereas $e_{t}$ is in the boundary of $M(S)$, since $t$ is an element of the boundary of $S$. This contradiction establishes $x y \in S x$ for every $y \in S^{0}$; since $S^{0}$ is dense in $S$ and $S x$ is closed, we have $x S \subseteq S x$ as desired.

(L) Let $\left\{y_{\alpha}\right\}_{\alpha \in D}$ be a net in $T$ converging to $y \in T$. Let $p \in(S y)^{0}=S^{0} y$. There exists $\delta \in D$ such that if $\alpha>\delta$, then $p \in\left(S y_{\alpha}\right)^{0}$. Let $2^{S}$ be the family of closed subsets of $S$, endowed with the Vietoris topology. It is well known that $2^{S}$ is a compact topological semigroup under the operation of set multiplication inherited from $S$. In particular, if $B$ denotes the cell boundary of $S$, we have $B y=\lim \sup \left\{B y_{\alpha}\right\}$ $=\lim \inf \left\{B y_{\alpha}\right\}$, as well as $S y=\lim \sup \left\{S y_{\alpha}\right\}=\lim \inf \left\{S y_{\alpha}\right\}$. Let $V$ be a connected open set containing $p$ (S is locally connected) such that $\bar{V} \subseteq S^{0} y$. Then there exists $\beta \in D$ such that if $\alpha>\beta$, then $B y_{\alpha} \subseteq(S \backslash \bar{V})$. Let $\gamma \in D$ such that if $\alpha>\gamma$, then $V \cap S y_{\alpha}$ $\neq \square$. Finally, let $\delta \in D$ such that $\delta>\beta, \gamma$. Then, for $\alpha>\delta$, we have $V \subseteq S y_{\alpha}$, since otherwise $B y_{\alpha}$ would separate $V$. Thus $p \in\left(S y_{\alpha}\right)^{0}$ for $\alpha>\delta$. It should be noted here that, although sequences are clearly sufficient to describe convergence in $S$, it is to our advantage later in the proof to have (L) stated for arbitrary nets.

In virtue of $(\mathrm{K})$ above, for each $g, x \in T$ there exists a unique $t \in T$ such that $g x=t g$; for fixed $g \in T$ this defines a map $F$ on $T$ into $T$ by $F(x)=t$ where $g x=t g$. By passing to the group $G$ it may be seen that $F$ is the restriction to $\varphi(T)$ of the inner automorphism generated by $\varphi(g)$, with the action translated back into $T$ by the function $\varphi^{-1}$. It follows immediately that $F$ is a topological isomorphism of $T$ into itself. Clearly $F$ admits a well-defined extension to $M(S)$ given by $F(e)=g e$ for each $e \in M(S)$. We permit the extended function to retain the name $F$.

(M) The function $F$ is a topological isomorphism of $S$ into $S$. From the remarks above, it remains to show that $F$ is one-to-one and continuous on $M(S)$. Suppose $g e=g f$, where $e, f \in M(S)$. Let $x \in$ Core $(e) \backslash\{1\}, y \in$ Core $(f) \mid\{1\}$. Let $s, t \in T$ such that $g x=t g, g y=s g$. Then $\operatorname{tg} e=g x e=g e$ and $s g e=s g f=g y f=g f=g e$, so that both $s$ and $t$ belong to Core $(g e)=$ Core $(g f)$. Now we have $s t=t s$, from which $g y x=s g x$ $=s t g=t s g=t g y=g x y$. By left cancellation, $y x=x y$ and therefore by part $(\mathrm{F})$ of the proof, $[x]=[y]$, whence $e=f$. This proves that $F$ is one-to-one on $M(S)$.

Continuity of $F$ on $T$ and $M(S)$ separately is immediate from the definition of $F$. Let $\left\{z_{n}\right\}$ be a sequence in $T$ converging to $e \in M(S)$. Let $\left\{F\left(z_{n}\right)\right\}$ cluster to $f$ in $S$. We show $f=g e=F(e)$. From the equalities $g z_{n}=F\left(z_{n}\right) g$ we have $g e=f g$; since $g e \in M(S)$ and $g \in T$, it follows from Theorem 3.1 that $f \in M(S)$, so that $f g=f$ and thus $g e=f$ as desired. Since $F$ restricted to $T$ is a homomorphism, $F$ is now a continuous iso- 
morphism of $S$ into itself. Since $S$ is compact, $F$ is also a closed map and hence a homeomorphism. This completes the proof of $(\mathrm{M})$.

(N) Let $g \in S^{0}$, and let $F$ be the function determined by $g$ as in (M) above. Then for each $x \in T$, we have $F(x) \notin(S x)^{0}=S^{0} x$. If $x=1$, this is clear. Otherwise, as in the argument for (K), let $A$ be the complement of $M(S)$ in the boundary of $S$, so that the boundary of $S x$ is $M(S) \cup A x$. From (F), the fixed point set of $S$ under $F$ is exactly the arc $[g]$; since $g \in S^{0}, e_{g} \in M(S)^{\circ}$. Hence $[g]$ meets each of the disjoint open sets $S \backslash S x$ (in 1) and $S x \backslash \bar{A} x$ (in $e_{g}$ ). Thus $[g] \cap \bar{A} x \neq \square$, whence $[g] \cap A x$ $\neq \square$. This proves that $F$ leaves a point of $A x$ fixed. On the other hand, were $F(x) \in S^{0} x$, we would have $F(T x)=F(T) F(x) \subseteq T\left(S^{0} x\right) \subseteq S^{0} x$, where the final inclusion follows from Lemma 2.2. This contradiction completes the proof.

(O) Let $g \in S^{0}$, and for each positive real number $r$, let $F_{r}$ be the function induced by $g^{r}$ as in the claims above. Note that, if $s$ is also a positive real number, then $F_{s+r}=F_{s} \cdot F_{r}$. Fix $x \in T$, and abbreviate $F_{r}(x)$ to $y_{r}$. Clearly $\left\{y_{r}\right\}$ forms a net directed by the natural order of $P$, the positive real numbers. We prove that the net $\left\{y_{r}\right\}$ converges to an element $y \in T \cap[g]$. Since this is immediate if $x=1$, assume $x \neq 1$. Let $z$ be any cluster point of $\left\{y_{r}\right\}$. To prove $z \in[g]$, it suffices to show $e_{g}=z e_{g}$. For each $r \in P, g^{r} x=y_{r} g^{r}$; since $\left\{g^{r}\right\}$ converges to $e_{g}$ and multiplication in $S$ is continuous, the desired equality holds. Now, if $z \in M(S)$, then $z=e_{g}$, whence $z \in M(S)^{0}$. The set $S x \mid \bar{A} x$ is therefore an open set in $S$ containing $z$, so there exists $r \in P$ such that $y_{r} \in S x \mid \bar{A} x$. Since $y_{r} \in T$, we have $F_{r}(x)=y_{r} \in S^{0} x$, which contradicts (N). Hence $z \in T$, and we have shown that any cluster point of $\left\{y_{r}\right\}$ belongs to $[g] \cap T$. To complete this claim, it must be proved that $\left\{y_{r}\right\}$ cannot cluster to two distinct elements $y$ and $z$ of $[g] \cap T$. Assume neither $y$ nor $z$ is 1 ; a suitable modification of the ensuing argument can be made easily to treat the case in which $\left\{y_{r}\right\}$ clusters to 1 . Set $z=y^{r}$, where, say, $r>1$, and set $p=y^{(r+1) / 2}$. Since $y \in S^{0}$, we have immediately that $p \in(S y)^{0}$ and $y^{r} \in(S p)^{0}$. Next, by chonsing a subnet of $\left\{y_{r}\right\}$ converging to $y$, we may apply part (L) of the proof to produce one positive real number $j$ such that $p \in S^{0} y_{j}$, so that $S p \subseteq S y_{j}$. Since $\left\{y_{r}\right\}$ also clusters to $z$, there exists a positive real number $k$ such that $k>j$ and $y_{k} \in(S p)^{0}$. Let $s=k-j$; then $F_{s}\left(y_{j}\right)=F_{s}\left(F_{j}(x)\right)=F_{s+j}(x)$ $=y_{k} \in(S p)^{0} \subseteq S y_{j}$, in contradiction to (N). This completes the proof of (O).

We are now prepared to extend the embedding function $\psi \varphi$ to $M(S)^{0}$. Recall, from part $(\mathrm{H})$, that a basis $\left\{x_{i}\right\}, i=1, \ldots, n$, has been fixed for $\mathscr{L}$ such that $\exp \left(x_{i}\right) \in \varphi\left(S^{0}\right)$ for $i=1, \ldots, n$. Fix $e \in M(S)^{0}$, and choose $g$ arbitrarily from Core $(e) \cap S^{0}$. As before, let $F_{r}$ be the function determined by $g^{r}$. We define the operator $\psi \varphi(e)$ by its action on the basis $\left\{x_{i}\right\}$. In particular, let $t_{i}$ $=\lim _{r \in P} F_{r}\left(\varphi^{-1}\left(\exp \left(x_{i}\right)\right)\right)$. Then, for any nonnegative real number $q$, we define $\left(\psi \varphi(e)\left(x_{i}\right)\right)(q)=\varphi\left(\left(t_{i}\right)^{q}\right)$. For negative $q$, we let $\left(\psi \varphi(e)\left(x_{i}\right)\right)(q)=\varphi\left(\left(t_{i}\right)^{-q}\right)^{-1}$. In this manner, it is clear that $\left(\psi \varphi(e)\left(x_{i}\right)\right)$ is a one parameter group in $G$ for $i=1, \ldots, n$. Further, the range of the operator $\psi \varphi(e)$ thus determined is the subspace of $\mathscr{L}$ spanned by $\exp ^{-1}(\varphi(g))$, which is proper in $\mathscr{L}$, so that $\psi \varphi(e)$ is not an invertible operator. As defined to the present, the operator $\psi \varphi(e)$ appears to be dependent on 
the choice of $g$ from Core $(e) \cap S^{0}$. The next lemma shows this not to be the case.

(P) Let $g \in S^{0}$, let $h=g^{t}$, where $t \in P$, and let $x \in T$. If $F_{r}$ is the function determined by $g^{r}$ as in $(\mathrm{M})$, then $\lim _{r \in P}\left\{F_{r}(x)\right\}=\lim _{r \in P}\left\{F_{t r}(x)\right\}$. This is clear, since either of these nets (directed by $P$ ) may be regarded as a subnet of the other.

(Q) The exponential function is a homeomorphism on $\exp ^{-1}\left(\varphi\left(S^{0}\right)\right)$. Let $c$ and $d$ be elements of $\exp ^{-1}\left(\varphi\left(S^{0}\right)\right)$, and suppose $\exp (c)=\exp (d)=\varphi(t) \in \varphi\left(S^{0}\right)$. Since $c$ is continuous and $\varphi\left(S^{0}\right)$ is open in $G$, there exists an open interval $U$ containing 1 such that $c(U) \subseteq\left(S^{0}\right)$. Let $a / b$ be a positive number in $U$. There exists $m \in S^{0}$ such that $c(a / b)=\varphi(m)$. Then $\varphi\left(m^{b}\right)=c(a)=(c(1))^{a}=\varphi\left(t^{a}\right)$, so that $m^{b}=t^{a}$ and thus $m=t^{a / b}$. It follows that, for all $r \in U$, we have $c(r)=\varphi\left(t^{r}\right)$. We have now shown that $c$ and the one parameter group $f$ defined by $f(r)=\varphi\left(t^{r}\right)$ if $r \geqq 0, f(r)$ $=\varphi\left(t^{-r}\right)^{-1}$ if $r<0$ agree on the open interval $U$. It is straightforward to prove that $c=f$ from this information. An analogous argument shows that $d=f$, whence $c=d$ and $(\exp )$ is one-to-one on $(\exp )^{-1}\left(\varphi\left(S^{0}\right)\right)$. Since $(\exp )^{-1}\left(\varphi\left(S^{0}\right)\right)$ is an open subset of Euclidean $n$-space and $\varphi\left(S^{0}\right)$ is homeomorphic to $n$-space, (exp) is a homeomorphism on $(\exp )^{-1}\left(\varphi\left(S^{0}\right)\right)$ by invariance of domain.

(R) For each $g \in S^{0}$, the function $\psi \varphi$ restricted to $[g]$ is a topological isomorphism. To see that this function is continuous at $e_{g}$, let $\left\{h_{n}\right\}$ be a sequence in $[g] \backslash\left\{e_{g}\right\}$ converging to $e_{g}$. Set $h_{n}=g^{r(n)}$; it is no loss of generality to assume the sequence $\{r(n)\}$ is monotonically increasing in $P$. It must be shown that, in the topology of $\mathscr{L},\left(\psi \varphi\left(h_{n}\right)\left(x_{i}\right)\right)$ converges to $\left(\psi \varphi\left(e_{g}\right)\left(x_{i}\right)\right)$ for $i=1, \ldots, n$. To this end, recall

$$
\begin{aligned}
\left(\psi \varphi\left(e_{g}\right)\left(x_{i}\right)\right)(1) & =\left(\lim _{r \in P} F_{r}\left(\varphi^{-1}\left(\exp \left(x_{i}\right)\right)\right)\right) \\
& =\lim _{r \in P}\left(\varphi\left(F_{r}\left(\varphi^{-1}\left(\exp \left(x_{i}\right)\right)\right)\right)\right)=\lim _{r \in P} \varphi\left(g^{r}\right) x_{i}(1) \varphi\left(g^{r}\right)^{-1} .
\end{aligned}
$$

On the other hand, $\left(\psi \varphi\left(h_{n}\right)\left(x_{i}\right)\right)(1)=\left(\varphi\left(g^{r(n)}\right) x_{i}(1)\right)\left(\varphi\left(g^{r(n)}\right)^{-1}\right)$. Hence, from part (O) we have $\exp \left(\psi \varphi\left(h_{n}\right)\left(x_{i}\right)\right)$ converges to $\exp \left(\psi \varphi\left(e_{g}\right)\left(x_{i}\right)\right)$. Further, since $g \in S^{0}$, $\exp \left(\psi \varphi\left(e_{g}\right)\left(x_{i}\right)\right) \in \varphi\left(S^{0}\right)$, which is open in $G$ so that, by (Q), $\left(\psi \varphi\left(h_{n}\right)\left(x_{i}\right)\right)$ converges to $\left(\psi \varphi\left(e_{g}\right)\left(x_{i}\right)\right)$. This completes the proof of $(\mathrm{R})$.

(S) For each $x \in S^{0} \cup M(S)^{0}$, $\operatorname{det}(\psi \varphi(x)) \in[0,1]$, where "det" is the determinant function. Furthermore, det $(\psi \varphi(x))=1$ if and only if $x=1$. This is immediate from part (R) above, together with the fact that the determinant function is a continuous homomorphism. Indeed, we have that (det $\psi \psi \varphi$ ) is a topological isomorphism of $[g]$ onto the real interval $[0,1]$ under real multiplication, for any $g \in S^{0}$.

(T) The function $\psi \varphi$ is continuous and one-to-one on $[S \backslash M(S)] \cup M(S)^{0}$. First, let $\left\{z_{m}\right\}$ be a sequence in $S \backslash M(S)$ converging to $e \in M(S)^{0}$, and let $g \in S^{0} \cap$ Core $(e)$. Let $x_{i}$ be a fixed arbitrary member of the previously mentioned basis of $\mathscr{L}$. It suffices to show that $\left\{\left(\psi \varphi\left(z_{m}\right)\right)\left(x_{i}\right)\right\}$ converges to $(\psi \varphi(e))\left(x_{i}\right)$; indeed, since $e \in M(S)^{0}$, $(\psi \varphi(e))\left(x_{i}\right) \in \exp ^{-1}\left(S^{0}\right)$. Thus, by (Q), we need only show $\left\{\exp \left(\left(\psi \varphi\left(z_{m}\right)\right)\left(x_{i}\right)\right)\right\}$ converges to $\exp \left((\psi \varphi(e))\left(x_{i}\right)\right)$. For each $m, \varphi\left(z_{m}\right)\left(x_{i}(1)\right) \varphi\left(z_{m}\right)^{-1}$ has the same determinant as $x_{i}(1)$; similarly, by the definition of $(\psi \varphi(e))$ we have $\operatorname{det}\left((\psi \varphi(e))\left(x_{i}\right)(1)\right)$ 
$=\operatorname{det}\left(x_{i}(1)\right)$. It now follows from the comments in (S) above that, if the sequence $\left\{\varphi\left(z_{m}\right)\left(x_{i}(1)\right) \varphi\left(z_{m}\right)^{-1}\right\}$ has all of its cluster points in $\varphi([g])$, then it must converge to $\left((\psi \varphi(e))\left(\left(x_{i}\right)(1)\right)\right)$. Let $t \in S^{0}$ such that $\varphi(t)=x_{i}(1)$, and let $F_{m}$ be the function generated by the equality $z_{m} s=F_{m}(s) z_{m}$ as in part (M) above. Since the sequence $\left\{z_{m} t\right\}$ converges to $e t=e$, we have that the sequence $\left\{F_{m}(t) z_{m}\right\}$ also converges to $e$. It now follows that, if $r$ is a cluster point in $S$ of $\left\{F_{m}(t)\right\}$, then $r e=e$, so that $r \in \operatorname{Core}(e)=[g]$. Finally, no cluster point of the sequence $\left\{F_{m}(t)\right\}$ can belong to $M(S)$, since det $\varphi(t)$ is not zero. Hence the sequence $\left\{\varphi\left(z_{m}\right)\left(x_{i}(1)\right) \varphi\left(z_{m}\right)^{-1}\right\}$ does cluster in $\varphi([g])$, and therefore it converges to the element $\left((\psi \varphi(e))\left(\left(x_{i}\right)(1)\right)\right)$.

To complete the proof of $(\mathrm{T})$, it remains to treat the case in which $\left\{e_{k}\right\}$ is a sequence in $M(S)^{0}$ converging to $e \in M(S)^{0}$. It suffices to show $\left\{\exp \left(\left(\psi \varphi\left(e_{k}\right)\right)\left(x_{i}\right)\right)\right\}$ clusters to $\exp \left((\psi \varphi(e))\left(x_{i}\right)\right)$. For each $k$, let $g_{k} \in S^{0} \cap$ Core $\left(e_{k}\right)$. For simplicity, let

$$
h_{k}=\lim _{n}\left(\varphi\left(g_{k}\right)\right)^{n} x_{i}(1)\left(\varphi\left(g_{k}\right)\right)^{-n}=\exp \left(\left(\psi \varphi\left(e_{k}\right)\right)\left(x_{i}\right)\right),
$$

and let

$$
h=\lim _{n}(\varphi(g))^{n} x_{i}(1)(\varphi(g))^{-1}=\exp \left((\psi \varphi(e))\left(x_{i}\right)\right),
$$

where $g \in S^{0} \cap$ Core $(e)$. Recall that $h_{k} \in \varphi\left(\left[g_{k}\right]\right), h \in \varphi([g])$, and note $\operatorname{det} h_{k}$ $=\operatorname{det} x_{i}(1)=\operatorname{det} h$ for each $k$. Let $s_{k} \in T$ such that $\varphi\left(s_{k}\right)=h_{k}$, and let $\left\{s_{k}\right\}$ cluster to $s$ in $S$. By the first part of the proof of (T), $s$ cannot belong to $M(S)^{0}$. On the other hand, since $s_{k} e_{k}=e_{k}$, we have by continuity that $s e=e$, whence $s \in \operatorname{Core}(e)$, so that $s \in$ Core $(e) \backslash\{e\}$. It follows that $\left\{h_{k}\right\}$ clusters to $\varphi(s)$. Further, since the determinant function is continuous, we have $\operatorname{det} \varphi(s)=\operatorname{det} h_{k}=\operatorname{det} h$. Hence, by part (R), $\varphi(s)$ $=h$. Thus $\left\{h_{k}\right\}$ clusters to $h$.

That $\psi \varphi$ is one-to-one on $M(S)^{0}$ is immediate from part $(\mathrm{O})$.

(U) The semigroup $S$ is topologically isomorphic to a subsemigroup of itself contained in $(S \backslash M(S)) \cup M(S)^{0}$. Fix $g \in S^{0}$, and let $F$ be the function defined before part (M) of the proof by $F(x)=t$ if $x \in T$ and $g x=\operatorname{tg}, F(e)=g e$ if $e \in M(S)$. Let $F_{n}$ be the function defined by $g^{n}$ in the analogous manner. By part (M) of the proof, each $F_{n}$ is a topological isomorphism of $S$ into $S$. We show there exists a positive integer $n$ such that $F_{n}(S) \subseteq(S \backslash M(S)) \cup M(S)^{0}$. Clearly, we need only produce $n$ such that $F_{n}(M(S)) \subseteq M(S)^{0}$. Let $g^{n}$ converge to $e_{g}$. Note $e_{g} \in M(S)^{0}$ by part (I). Hence, for each $q \in M(S)$, there exists a positive integer $n(q)$ such that $g^{n(q)} \cdot q \in M(S)^{0}$. By continuity of multiplication, there exists a set $V_{q}$ open in $M(S)$, containing $q$ such that $g^{n(q)} \cdot V_{q} \subseteq M(S)^{0}$. Let $\left\{V_{q(i)}\right\}, i=1, \ldots, b$, be a finite cover of $M(S)$ determined in this manner, and let $n=\max \{n(q(i)): i=1, \ldots, b\}$. Thus, if $q \in M(S)$, let $q \in V_{q(i)}$. Then $g^{n} q=g^{n-n(q(i))} g^{n(q(i))} q \in g^{n-n(q(i))} M(S)^{0}$. By part (J) above, the latter set is a subset of $M(S)^{0}$. Hence $g^{n} M(S) \subseteq M(S)^{0}$, whence $F_{n}(S) \subseteq(S \backslash M(S)) \cup M(S)^{0}$.

The proof of Theorem 4.1 is completed by noting that the composite function $(\psi \varphi) \cdot F_{n}$ is a continuous isomorphism of the compact semigroup $S$ into $M_{n}(R)$, and is hence a topological isomorphism. 


\section{REFERENCES}

1. J. T. Borrego, H. Cohen and E. E. DeVun, Uniquely representable semigroups, Pacific J. Math. (to appear).

2. D. R. Brown, Matrix representations of compact simple semigroups, Duke Math. J. 33 (1966), 69-73. MR 32 \#5786.

3. D. R. Brown and M. Friedberg, Representation theorems for uniquely divisible semigroups, Duke Math. J. 35 (1968), 341-352. MR 36 \#6532.

4. - A new notion of semicharacters, Trans. Amer. Math. Soc. 141 (1969), 387-401. MR 39 \#4311.

5. D. R. Brown and J. G. LaTorre, A characterization of uniquely divisible commutative semigroups, Pacific J. Math. 18 (1966), 57-60. MR 34 \#261.

6. Francis T. Christoph, On topological semigroups, Dissertation, Rutgers University, New Brunswick, N. J., 1969.

7. A. H. Clifford and G. B. Preston, The algebraic theory of semigroups. Vol. I, Math. Surveys, no. 7, Amer. Math. Soc., Providence, R. I., 1961. MR 24 \#A2627.

8. Robert Ellis, Locally compact transformation groups, Duke Math. J. 24 (1957), 119-125. MR 19, 561.

9. M. Friedberg, On representations of certain semigroups, Pacific J. Math. 19 (1966), 269-274. MR 34 \#2764.

10. J. A. Hildebrant, On compact unithetic semigroups, Pacific J. Math. 21 (1967), 265-273. MR 35 \#2992.

11. - On uniquely divisible semigroups on the two-cell, Pacific J. Math. 23 (1967), 91-95. MR 35 \#4329.

12. K. H. Hofmann and P. S. Mostert, Elements of compact semigroups, C. E. Merrill, Columbus, Ohio, 1966. MR 35 \#285.

13. J. G. Horne, Semigroups on a half-space, Trans. Amer. Math. Soc. 147 (1970), 1-53.

14. Anne Lester Hudson, Some semigroups on the two-cell, Proc. Amer. Math. Soc. 10 (1959), 648-655. MR 21 \#5698.

15. Witold Hurewicz and Henry Wallman, Dimension theory, Princeton Math. Series, vol. 4, Princeton Univ. Press, Princeton, N. J., 1941. MR 3, 312.

16. Klaus Keimel, Eine Exponentialfunktion für kompakte abelsche Halbgruppen, Math. Z. 96 (1967), 7-25. MR 34 \#2765.

17. - Lokal kompakte Kegelhalbgruppen und deren Einbettung in topologische Vektorräume, Math. Z. 99 (1967), 405-428.

18. R. J. Koch, On monothetic semigroups, Proc. Amer. Math. Soc. 8 (1957), 397-401. MR $19,290$.

19. Deane Montgomery and Leo Zippin, Topological transformation groups, Interscience, New York, 1955. MR 17, 383.

20. Leopoldo Nachbin, Topology and order, Van Nostrand Math. Studies, no. 4, Van Nostrand, Princeton, N. J., 1965. MR 36 \#2125.

21. R. C. Phillips, Interval clans with nondegenerate kernel, Proc. Amer. Math. Soc. 14 (1963), 396-400. MR 26 \#5547.

22. Neal J. Rothman, Embedding of topological semigroups, Math. Ann. 139 (1960), 197-203. MR 22 \#6871.

UNIVERSITY OF HOUSTON, Houston, TeXas 77004 